\title{
Bulk spectral functions in single and multi-scalar gravity duals
}

\author{
Todd Springer ${ }^{*}$ Charles Gale ${ }^{\dagger}$ and Sangyong Jeon ${ }^{\dagger}$ \\ Department of Physics, McGill University, Montreal, Quebec, H3A2T8, Canada
}

(Dated: December 22, 2010)

\begin{abstract}
We examine two point correlation functions involving the trace of the energy momentum tensor in five-dimensional dual gravity theories supported by one or more scalar fields. A prescription for determining bulk channel spectral functions is developed. This prescription generalizes previous work which centered on one scalar field. As an application of these techniques, we investigate the bulk spectral function and corresponding sum rule in the Chamblin-Reall background. We show that, when expressed in terms of the beta function, the sum rule for the Chamblin-Reall background can be written in a form similar to the sum rule in Yang-Mills theory.
\end{abstract}

\section{INTRODUCTION}

In recent years, there has been great interest in the correspondence between certain strongly coupled gauge theories and extra-dimensional gravity theories. This duality, originally inspired by the AdS/CFT (Anti deSitter/Conformal Field Theory) correspondence [1-4 has led to a set of tools which allow for the computation of physical observables in some strongly coupled theories. These tools have renewed interest in the possibility of a nonperturbative description of QCD and the strongly coupled matter created in heavy ion collisions, the QGP (quark-gluon plasma). Many excellent reviews on the vast literature of this subject are now available, among them are [5, 9].

In order to describe theories which appear in the real world, such as QCD, one must move beyond scale invariant theories to nonconformal ones. There are several known examples of string theory setups which give rise to a dual nonconformal field theory [10 13. An alternative, phenomenologically based approach involves constructing an effective five-dimensional gravity background and assuming that it has a dual gauge theory description. It is also assumed that all of the AdS/CFT machinery still works for this phenomenological setup. The simplest of such backgrounds involve Einstein gravity, and one or more interacting scalar fields. Such models have been studied extensively in the literature [14 24]. To date, most phenomenological models have included a single scalar field for simplicity. However, it seems necessary to include two scalar fields to properly describe both conformal and chiral symmetry breaking [22, 25, 26]. Furthermore, many string theoretical setups can be reduced to effective five-dimensional backgrounds supported by multiple scalar fields [27, 28]

In this work, we will use such multi-scalar gravity theories to study two point functions of the energymomentum tensor in the dual thermal gauge theory. Such correlation functions are interesting because they

\footnotetext{
* springer@physics.mcgill.ca

$\dagger$ gale@physics.mcgill.ca

$\ddagger \frac{\text { jeon@physics.mcgill.ca }}{\text { jen }}$
}

allow for the computation of transport coefficients (like shear and bulk viscosities). Transport coefficients can be read off from the low energy behavior of the spectral density (the imaginary part of these correlation functions). These transport coefficients are useful in hydrodynamic descriptions of the QGP, and are not otherwise calculable perturbatively since the QGP is strongly coupled near the phase transition. In addition to AdS/CFT, there have been attempts to extract transport coefficients from the lattice [29 31]. Typically one needs to include an ansatz for the spectral density in order to perform such an extraction ${ }^{1}$ In this case, insight gained from gauge/gravity duality into the behavior of spectral functions at strong coupling could be useful.

A prescription for determining the bulk spectral density in a single-scalar gravity dual was given in [14. One of the purposes of the current work is to generalize this prescription to a matter sector which includes multiple scalar fields. The results of 14 employed a coordinate system where the scalar field was identified with the radial coordinate. While this simplified the analysis, it made the generalization to multiple scalar fields difficult. In the present work, we will choose our coordinates and gauges differently so that generalization to multiple scalar fields is straightforward. It should be noted that a different method for determining the bulk viscosity in multi-scalar gravity dual theories was developed recently in 33, however our method allows for the calculation of the spectral function at all values of the frequency $w$.

As an example application, we will determine the bulk spectral density in a particular single scalar setup called the Chamblin-Reall background 34. This background is one of the only known nonconformal setups where exact results for the bulk viscosity are calculable analytically. We emphasize that this model is not particularly well suited for QGP phenomenology; it has no conserved charge, and also has the peculiar feature of both being nonconformal and having a speed of sound which is independent of temperature. However, recently it was found that the dynamics of a more complicated string

\footnotetext{
1 See 32 for a critical examination of the methodology of such an extraction.
} 
theory setup were captured by an effective single scalar Chamblin-Reall background 35. This may be an indication that there is a nontrivial connection between the Chamblin-Reall background and nonconformal deformations of more sophisticated string theory backgrounds.

While the thermodynamics and transport coefficients of this model have already been studied in the literature [15, 20, 21, 36 39, the full bulk spectral density (at nonzero frequency) has not been previously presented, and we will use the methods developed in the first part of this paper to do so here. (This background does not utilize the full power of our methods as it only contains one scalar field; however, by working in this model we will be able to perform consistency checks with previous results.)

Once we have determined the bulk spectral density, we take the opportunity to investigate the bulk sum rule of [40] in the context of this model. We notice an intriguing fact that for the Chamblin-Reall background, the shear and bulk spectral densities are simply related at all frequencies. We also demonstrate that the bulk sum rule in this model does not agree with the bulk sum rule written down in [40] and we explain the reason for the discrepancy.

Recently, a thorough study of the shear correlation function and sum rule in the Chamblin-Reall model was completed in 41. The latter half of the present work extends this analysis to the bulk sector.

The paper is organized as follows. In Sec. III we detail the multi-scalar gravity dual in which we are working, and set up the relevant perturbations which need to be analyzed in order to calculate the two point functions. In Secs. III and IV, we generalize the work of 14 by detailing our prescription for calculating the bulk spectral density, $\rho^{\text {bulk }}$ in a multi-scalar gravity dual. This involves the on-shell gravitational action which is given in Sec. III and the solution of relevant equations for the perturbations given in Sec. IV] Once we have developed our method for calculating the bulk spectral density, we apply it to the case of the Chamblin-Reall background in Sec. V] We provide explicit results for the spectral density, Euclidean correlation functions, and we also derive a bulk sum rule in this model. We summarize our main conclusions and discuss some possible directions for future work in Sec. VI. Finally, we provide some of the technical details of the calculations as well as some useful reference formulae in the appendices.

\section{BACKGROUND AND PERTURBATIONS}

In this work, we will be interested in the retarded correlation functions of a strongly coupled, nonconformal field theory,

$$
G_{R}^{\mu \nu \alpha \beta}(w) \equiv-i \int d^{4} x e^{i w t}\left\langle\left[T^{\mu \nu}(x), T^{\alpha \beta}(0)\right]\right\rangle \theta(t),
$$

with associated spectral densities

$$
\rho^{\mu \nu \alpha \beta}(w) \equiv-\operatorname{Im} G_{R}^{\mu \nu \alpha \beta}(w) .
$$

We will use the labels

$$
\begin{aligned}
G_{R}^{\text {shear }} & \equiv G_{R}^{x y x y}, \\
G_{R}^{\text {bulk }} & \equiv \eta_{\mu \nu} \eta_{\alpha \beta} G_{R}^{\mu \nu \alpha \beta},
\end{aligned}
$$

and similarly for the spectral functions. Here $\eta_{\mu \nu}$ denotes the four dimensional Minkowski metric. Kubo formulas relate the low energy limit of the spectral functions to the shear and bulk viscosities: $\eta$ and $\zeta$ respectively

$$
\begin{aligned}
& \eta=\lim _{w \rightarrow 0} \frac{\rho^{\text {shear }}(w)}{w}, \\
& \zeta=\frac{1}{9} \lim _{w \rightarrow 0} \frac{\rho^{\text {bulk }}(w)}{w} .
\end{aligned}
$$

Often in this work, we will be interested in quantities which have their vacuum (zero-temperature) part subtracted. We use the symbol $\Delta$ to denote this subtraction. For example,

$$
\Delta G_{R}(w) \equiv G_{R}(w)-G_{R}(w)_{T=0} .
$$

We will compute the correlation functions of interest using tools provided by AdS/CFT by working in a dual gravitational theory. The dual gravitational theory under consideration is a five-dimensional multi-scalar theory,

$$
\begin{aligned}
\mathcal{S} & =\frac{1}{2 \kappa} \int d^{5} x \sqrt{-g}\left[R-\frac{1}{2} \partial_{\mu} \phi_{a} \partial^{\mu} \phi_{a}-V\left(\phi_{1}, \ldots \phi_{n}\right)\right] \\
& +\frac{1}{\kappa} \int d^{4} x \sqrt{-\gamma} \theta
\end{aligned}
$$

Here, $\kappa$ is related to the five-dimensional Newton's constant, $\kappa=8 \pi G_{5}$. Summation is implied over the index " $a$ " which labels each scalar field. This index runs from 1 to $n$ with $n$ denoting the total number of scalar fields. These scalar fields in the bulk correspond to operators in the dual field theory.

The second term in (8) is a boundary term (the Gibbons-Hawking term). In this term, $\gamma_{\mu \nu}$ is the induced metric at the boundary, $\theta_{\mu \nu}$ is the second fundamental form

$$
\theta_{\mu \nu}=\nabla_{\mu} \hat{N}_{\nu}
$$

the unit vector normal to the boundary is denoted by $\hat{N}^{\mu}$, and $\nabla_{\mu}$ is the covariant derivative with respect to the background metric.

In order to access two point correlation functions in the dual field theory, one must add perturbations to the background.

$$
\begin{aligned}
g_{\mu \nu} & \longrightarrow g_{\mu \nu}+h_{\mu \nu} \\
\phi_{i} & \longrightarrow \Phi_{i}+\varphi_{i} .
\end{aligned}
$$

The quantities $g_{\mu \nu}$ and $\Phi_{i}$ are the background fields, and $h_{\mu \nu}$ and $\varphi_{i}$ are the perturbations. The metric perturbations are generally classified under $S O(2)$ rotations in 
the $x_{1}, x_{2}$ plane, with the momentum $\vec{q}$ pointing in the $x_{3}$ direction [14, 42, 43. The bulk mode (which is also sometimes called the "sound mode" in the literature) is the scalar mode containing perturbations which are invariant under such rotations.

In this work, we will be interested in correlation functions at zero spatial momentum $\vec{q}$, and thus instead of $S O(2)$ rotations, we have the full $S O(3)$ symmetry. There are four metric perturbations which fulfill the requirement of rotational invariance: $h_{00}, h_{i}^{i}, h_{05}$, and $h_{55}$. Latin indices on the metric components are assumed to run over the spatial dimensions $i=1,2,3$. There are also $n$ perturbations, one for each scalar field $\varphi_{a}$.

Not all of these perturbations are physical due to freedom under diffeomorphisms:

$$
\begin{aligned}
h_{\mu \nu} & \rightarrow h_{\mu \nu}-\nabla_{\mu} \psi_{\nu}-\nabla_{\nu} \psi_{\mu} \\
\varphi_{i} & \rightarrow \varphi_{i}-\psi^{\mu} \partial_{\mu} \Phi_{i}
\end{aligned}
$$

for any vector $\psi^{\mu}$. (Unfortunately, this is often called "gauge freedom", though it is not the same as gauge freedom in field theory). In 14, only one scalar field was considered, and the gauge was chosen so that perturbations $\varphi$ and $h_{05}$ vanished. In this work, we will employ the radial gauge $\left(h_{05}=h_{55}=0\right)$ with perturbations $h_{0}^{0}, h_{i}^{i}$, and $\varphi_{a}$ nonvanishing. There are two reasons for this choice of gauge over that of [14]. First, doing the computation in another gauge allows for consistency checks between the two calculations. Secondly, and more importantly, the choice of the radial gauge allows one to rather easily generalize the computation to multiple scalar fields. Such a generalization has not been previously presented, and it is one of the central results of the present work.

We define the background and perturbations as

$$
\begin{aligned}
d s^{2} & =g_{t t}(z)[1+A(t, z)] d t^{2} \\
& +g_{x x}(z)[1+B(t, z)] d \mathbf{x}^{2}+g_{z z}(z) d z^{2}, \\
\phi_{i}(t, z) & =\Phi_{i}(z)+\varphi_{i}(t, z) .
\end{aligned}
$$

The vector $\vec{x}$ spans the usual 3 -spatial coordinates. The coordinate $z$ labels the "extra" radial dimension; the dual four dimensional field theory exists on the boundary at $z=0$. The background is assumed to be static, and all time dependence is contained in the perturbations $A, B, \varphi_{i}$.

The radial gauge is initially chosen for the purposes of writing down the relevant equations and on-shell action. However, it is desirable to ultimately work in terms of gauge invariant perturbations, which are invariant under infinitesimal diffeomorphisms [20, 43, 44. In [20, the more general case of perturbations which depend on both space and time was examined. In this more general case, it was shown that there are $n+1$ gauge invariant variables where $n$ is the number of scalar fields in the theory. These variables were denoted as $\mathcal{Z}_{0}$ and $\mathcal{Z}_{\Phi i}$. Here, we are interested in the special case where the perturbations do not depend on the three spatial coordinates; in this case, the variable $\mathcal{Z}_{0}$ vanishes. We are left with $n$ gauge invariant variables, one for each scalar degree of freedom. These variables have the form:

$$
\mathcal{Z}_{\Phi i}(z)=\varphi_{i}(z)-\frac{\Phi_{i}^{\prime}(z) g_{x x}(z)}{g_{x x}^{\prime}(z)} B(z) .
$$

Primes denote derivatives with respect to the radial coordinate $z$. It is straightforward to check that these variables do not transform under the diffeomorphisms (12) and (13).

At first, it is perhaps surprising that the perturbation $A$ (which is dual to the operator $T^{00}$ in the gauge theory) cannot be put into a gauge invariant variable. In fact, this behavior is consistent with the expected structure of the correlators at zero spatial momentum, as we will now show. Because $A$ cannot be put into a gauge invariant variable, all correlation functions which involve $T^{00}$ vanish (up to contact terms) in the limit of zero spatial momentum. The vanishing of spectral functions involving $T^{00}$ is expected at $\vec{q}=0$ on general grounds. A general correlation function of components of the energy momentum tensor can be defined to satisfy the Ward identity [42, 43]

$$
q_{\mu} G^{\mu \nu \alpha \beta}=\text { contact terms. }
$$

The contact terms which appear on the right hand side of this equation depend on how the correlation function $G$ is defined. (Note that $G$ here does not necessarily coincide with the retarded correlation function $G_{R}$ defined in (1)). Here, the only necessary detail concerning the contact terms is that they are all real, so that regardless of the definition of the correlation function, the spectral density is the same as that given in (2). The Ward identity implies that the spectral density is transverse:

$$
q_{\mu}\left[\operatorname{Im} G^{\mu \nu \alpha \beta}\right]=-q_{\mu} \rho^{\mu \nu \alpha \beta}=0 .
$$

Writing this in components and using the definition of the four-momentum $q^{\mu}=(w, \vec{q})$,

$$
w \rho^{0 \nu \alpha \beta}=q_{i} \rho^{i \nu \alpha \beta} .
$$

Taking the limit where $\vec{q} \rightarrow 0$, the right side vanishes because the correlation functions are nonsingular functions at vanishing spatial momentum ${ }^{2}$ Then, we see that

$$
\rho^{0 \nu \alpha \beta}(w, \vec{q} \rightarrow 0)=0 .
$$

Our calculations in the dual gravity theory will be consistent with this result.

\section{ON-SHELL ACTION}

The prescription for calculating Minkowski space two point functions of a strongly coupled field theory from

\footnotetext{
2 This is true except in some very special cases where excitations at $w \neq 0$ do not decay as a function of time 45, 46]
} 
gauge/gravity duality was first given in [47. One needs to solve the linearized Einstein equations for the perturbations, and plug the results back into the action which has been expended to quadratic order in the perturbations. In this section we will expand the action and write it in terms of the gauge invariant variables. The main result of this section is an expression for the spectral densities in terms of the gauge invariant variables.

Let us assume that we can write our background metric in the coordinate system

$$
d s^{2}=g_{x x}(z)\left[-f(z) d t^{2}+d \vec{x}^{2}+\frac{d z^{2}}{f(z)}\right] .
$$

This coordinate system is only chosen as a calculational aid for the moment. We shall see that our final results will be valid in any coordinate system. With the use of the background equations of motion, and the linearized equations for the perturbations one can write the on-shell action, expanded to quadratic order in the perturbations, in the form

$$
\begin{aligned}
\mathcal{S}_{2} & =\frac{-V_{3}}{4 \kappa} \int \frac{d w}{2 \pi} g_{x x}^{3 / 2} f\left[\mathcal{Z}_{\Phi a}(-w, z) \mathcal{Z}_{\Phi a}^{\prime}(w, z)\right. \\
& \left.+Q(w, z)^{T} \xi(z) Q(-w, z)\right] .
\end{aligned}
$$

A derivation of this equation is presented in Appendix $\mathrm{B}$. The perturbations depend on $w$ rather than $t$ now because we are working with the Fourier transform. A summation over the repeated index $a$ (which takes values from 1 to $n$ ) is implied. Here, $V_{3}$ denotes the 3 -volume (integration over the three spatial coordinates), $Q$ is a vector made up of perturbations,

$$
Q(w, z)=\left(\begin{array}{c}
A(w, z) \\
B(w, z) \\
\varphi_{1}(w, z) \\
\vdots \\
\varphi_{n}(w, z)
\end{array}\right)
$$

and $\xi$ is a symmetric $(2+\mathrm{n}) \times(2+\mathrm{n})$ matrix, the components of which are unimportant for our present purposes (but are given in Appendix B). This expression for the on-shell action does not contain possible counter terms that are necessary to regulate UV divergences. We do not need to consider such counter terms, because the UV divergences generally only affect the real part of the onshell action [14, 47, 48. In this work, we will only be interested in the spectral functions, which are computed from the imaginary part of the on-shell action. In other words, regularizing counter terms will change the components of the matrix $\xi$, but this matrix does not contribute to the spectral functions.
The imaginary part of the on-shell action can be written

$$
\begin{aligned}
\operatorname{Im} \mathcal{S}_{2} & =\frac{T s V_{3}}{4 i} \int \frac{d w}{2 \pi}\left(\frac{g_{x x}(z)}{g_{x x}\left(z_{h}\right)}\right)^{3 / 2} \frac{f(z)}{f^{\prime}\left(z_{h}\right)} \\
& \times\left[\mathcal{Z}_{\Phi a}^{\prime}(w, z) \mathcal{Z}_{\Phi a}(-w, z)-(w \rightarrow-w)\right] .
\end{aligned}
$$

Here the temperature $T$ and entropy density $s$ have been used (details can be found in Appendix B. It is well known that the imaginary part of the on-shell action is proportional to a conserved quantity (see [14, 47, 48]), and thus we can evaluate the above expression at any value of $z$. We choose to evaluate it at the horizon, and make use of the incoming wave condition

$$
\mathcal{Z}_{\Phi i}^{\prime}\left(z_{h}\right)\left(z-z_{h}\right)=\frac{-i w}{4 \pi T} \mathcal{Z}_{\Phi i}\left(z_{h}\right)\left[1+\mathcal{O}\left(z-z_{h}\right)\right]
$$

and the near-horizon behavior of the metric

$$
\left.\frac{f^{\prime}(z)}{f(z)}\right|_{z \rightarrow z_{h}}=\frac{1}{z-z_{h}}+\mathcal{O}(1)
$$

The result is

$$
-\operatorname{Im} \mathcal{S}_{2}=\frac{s V_{3}}{8 \pi} \int \frac{w d w}{2 \pi}\left[\mathcal{Z}_{\Phi a}\left(w, z_{h}\right) \mathcal{Z}_{\Phi a}\left(-w, z_{h}\right)\right]
$$

which can be written

$$
\begin{aligned}
& -\operatorname{Im} \mathcal{S}_{2}= \\
& \sum_{a=1}^{n} \frac{s V_{3}}{8 \pi} \int \frac{w d w}{2 \pi} \mathcal{Z}_{\Phi a}^{0}(-w)\left[\frac{\mathcal{Z}_{\Phi a}^{h}(w) \mathcal{Z}_{\Phi a}^{h}(-w)}{\mathcal{Z}_{\Phi a}^{0}(-w) \mathcal{Z}_{\Phi a}^{0}(w)}\right] \mathcal{Z}_{\Phi a}^{0}(w)
\end{aligned}
$$

We have chosen to write it in this form because the quantity in square brackets is most directly related to the spectral density. The superscripts " 0 " and "h" denote the boundary and horizon value of the perturbations at $z \rightarrow 0$ and $z \rightarrow z_{h}$. Often the boundary values are divergent (though the final answer will not be). To be precise, one should introduce a UV regulator $\epsilon$

$$
\begin{aligned}
& \mathcal{Z}_{\Phi a}^{0} \equiv \mathcal{Z}_{\Phi a}(w, \epsilon), \\
& \mathcal{Z}_{\Phi a}^{h} \equiv \mathcal{Z}_{\Phi a}\left(w, z_{h}\right),
\end{aligned}
$$

which is assumed to be small. At the end of the calculation one should take $\epsilon \rightarrow 0$.

We will need to use the fact that due to the definition of $\mathcal{Z}_{\Phi i}$ 


$$
\mathcal{Z}_{\Phi i}^{0}(-w) \mathcal{Z}_{\Phi i}^{0}(w)=\left[Q^{0}(-w)\right]^{T}\left(\begin{array}{cccccc}
1 & 2 & \ldots & 2+i & \ldots & 2+n \\
0 & 0 & \ldots & 0 & \ldots & 0 \\
0 & k_{i}(\epsilon)^{2} & \ldots & -k_{i}(\epsilon) & \ldots & 0 \\
\vdots & \vdots & \ddots & \vdots & \vdots & \vdots \\
0 & -k_{i}(\epsilon) & \ldots & 1 & \ldots & 0 \\
\vdots & \vdots & \ldots & \vdots & \ddots & \vdots \\
0 & 0 & \cdots & 0 & \cdots & 0
\end{array}\right) Q^{0}(w) \text { (No sum over i), }
$$

where

$$
k_{i}(z) \equiv \frac{\Phi_{i}^{\prime}(z) g_{x x}(z)}{g_{x x}^{\prime}(z)}
$$

We have added column labels above the matrix to aid the reader. The matrix is square, so the same labels apply to the rows. To be clear, all ellipses (...) in the above matrix denote zeros; the nonvanishing elements of this matrix are written explicitly here.

The Son and Starinets prescription for the correlation functions [4], (see also [8] for a nice treatment of mixed operators) can be stated as follows. After writing the onshell action in the form

$$
\operatorname{Im} \mathcal{S}_{2}=\int \frac{d w}{2 \pi}\left[Q^{0}(-w)\right]^{T} M Q^{0}(w)
$$

the spectral density matrix is

$$
\rho(w)=-2 M
$$

For simplicity, we define

$$
R_{i}(w, \epsilon) \equiv\left[\frac{\mathcal{Z}_{\Phi i}^{h}(w) \mathcal{Z}_{\Phi i}^{h}(-w)}{\mathcal{Z}_{\Phi i}^{0}(-w) \mathcal{Z}_{\Phi i}^{0}(w)}\right]=\frac{\left|\mathcal{Z}_{\Phi i}^{h}(w)\right|^{2}}{\left|\mathcal{Z}_{\Phi i}^{0}(w)\right|^{2}}
$$

The repeated index $i$ is not summed, and the second equality is due to the fact that $\mathcal{Z}(w)^{*}=\mathcal{Z}(-w)$. Combining (28), (31), (34), and (35) we have the main result of this section:

$$
\rho(w)=\frac{s w}{4 \pi} \lim _{\epsilon \rightarrow 0}\left(\begin{array}{ccccc}
\mathcal{O}^{A} & \mathcal{O}^{B} & \mathcal{O}^{\Phi_{1}} & \ldots & \mathcal{O}^{\Phi_{n}} \\
\cline { 2 - 6 } 0 & 0 & 0 & \ldots & 0 \\
0 & \sum_{a=1}^{n} k_{a}(\epsilon)^{2} R_{a} & -k_{1}(\epsilon) R_{1} & \ldots & -k_{n}(\epsilon) R_{n} \\
0 & -k_{1}(\epsilon) R_{1} & R_{1} & \ldots & 0 \\
\vdots & \vdots & \vdots & \ddots & \vdots \\
0 & -k_{n}(\epsilon) R_{n} & 0 & \ldots & R_{n}
\end{array}\right) .
$$

The ellipses in this matrix do not necessarily denote zeros. To clarify, the entries in the second row and the second column of this matrix are all nonvanishing except for the zero which is written explicitly above. Similarly, the entries on the diagonal are all nonvanishing, except the upper left corner. All other entries are zero. The column labels are to aid the reader in distinguishing the significance of each entry; the matrix is square so the same labels apply to the rows.

This matrix contains all information about spectral densities of the relevant operators in the dual field theory. For example, suppose one is interested in the spectral density of the two point function $\left\langle\mathcal{O}^{B} \mathcal{O}^{\Phi_{1}}\right\rangle$ for two operators $\mathcal{O}^{B}$ and $\mathcal{O}^{\Phi_{1}}$ which are dual to the fields $B$ and $\Phi_{1}$. The examination of the above matrix tells us the answer is

$$
\rho^{B \phi_{1}}(w)=-\lim _{\epsilon \rightarrow 0} \frac{s w}{4 \pi} k_{1}(\epsilon) R_{1}(w, \epsilon) .
$$

Note that all spectral functions involving the operator $\mathcal{O}_{A} \sim T^{00}$ vanish. This is the expected behavior at zero spatial momentum as explained in Sec. III]

In this work, our primary interest is $\rho^{\text {bulk }}$. The operator dual to the perturbation $B$ is $\frac{1}{2} T_{i}^{i}$ (the factor of a half introduces an extra factor of 4 in the correlation function) [42. Thus, we need the matrix element in the second row, second column:

$$
\rho^{\mathrm{bulk}}(w)=\frac{s w}{\pi} \lim _{\epsilon \rightarrow 0} \sum_{a=1}^{n} k_{a}(\epsilon)^{2} R_{a}(w, \epsilon) .
$$

If one is only interested in the bulk viscosity, one can apply the Kubo formula (6),

$$
\zeta=\frac{s}{9 \pi} \lim _{\epsilon \rightarrow 0} \sum_{a=1}^{n} k_{a}(\epsilon)^{2} R_{a}(0, \epsilon) .
$$

It is worth emphasizing that the $k_{i}$ functions do not change under redefinition of the radial coordinate. Cer- 
tainly the bulk spectral density and bulk viscosity which are physical quantities must not depend on the choice of coordinates in the gravity dual. Thus, the $R_{a}$ functions must also be coordinate independent (we will be able to see this explicitly in the next section). Therefore, this prescription for calculating the spectral density and bulk viscosity is valid in any background coordinate system.

In order to evaluate these quantities one must compute the $R_{i}$ functions by solving the linearized equations for the gauge invariant fluctuations $\mathcal{Z}_{\Phi i}$. In the next section we will write down the relevant equations.

\section{LINEARIZED EQUATIONS}

In the previous section, we gave results for the bulk spectral function (and bulk viscosity) in terms of gauge invariant perturbations. In order to get an explicit result, one must solve the linearized Einstein equations for the perturbations. We will now detail the equations which need to be solved.

\section{A. Theories with multiple scalars}

In [20], sound mode perturbations were examined for a general, multi-scalar gravity dual with an arbitrary number of spatial dimensions denoted by $p$. The equations for the gauge invariant variables were given in full generality, assuming both space and time dependence. In the general case there is another gauge invariant variable $\mathcal{Z}_{0}$ which appears in addition to the $\mathcal{Z}_{\Phi i}$ which have already been introduced in the previous section. The equation that is relevant for our purposes is $3^{3}$

$$
\begin{aligned}
& \frac{g_{z z}}{\sqrt{-g}} \partial_{z}\left[\sqrt{-g} g^{z z} \mathcal{Z}_{\Phi i}^{\prime}\right]-\mathcal{Z}_{\Phi i} g_{z z}\left(w^{2} g^{t t}+q^{2} g^{x x}\right) \\
- & \frac{2 k_{i}}{p} \sum_{a=1}^{n} \mathcal{Z}_{\Phi a} \Phi_{a}^{\prime}\left[\mathcal{D}_{L}\left[\sqrt{-g} g^{z z} \Phi_{a}^{\prime}\right]+\frac{p k_{i}^{\prime}}{\alpha k_{i}}\left(q^{2}-\frac{w^{2}}{f}\right)\right] \\
- & g_{z z} \sum_{a=1}^{n} \mathcal{Z}_{\Phi a} \frac{\partial^{2} V}{\partial \Phi_{i} \partial \Phi_{a}}+\frac{2 k_{i}^{\prime}}{\alpha \sqrt{f}} \partial_{z}\left[\frac{\mathcal{Z}_{0}}{\sqrt{f}}\right]=0
\end{aligned}
$$

Here, the notation $\mathcal{D}_{L}$ denotes the logarithmic derivative

$$
\mathcal{D}_{L}[Y(z)]=Y^{\prime}(z) / Y(z),
$$

and the quantity $\alpha$ is defined as

$$
\alpha(z) \equiv q^{2}\left((p-1)+\frac{\mathcal{D}_{L}\left[g_{t t}\right]}{\mathcal{D}_{L}\left[g_{x x}\right]}\right)-\frac{p w^{2}}{f} .
$$

In the current work, we will limit ourselves to the case of zero spatial momentum $q=0$, and three spatial dimensions $p=3$. When the spatial momentum of the

\footnotetext{
3 This is Eq. (66) in [20]. Readers should note a different definition of the function $f$ in this paper. We have re-written the equation to correspond with the definitions given in the present work.
}

perturbations is set to zero, the gauge invariant variable $\mathcal{Z}_{0}$ vanishes identically. Hence, this equation simplifies to

$$
\begin{gathered}
\frac{1}{\sqrt{-g}} \partial_{z}\left[\sqrt{-g} g^{z z} \mathcal{Z}_{\Phi i}^{\prime}\right]-w^{2} g^{t t} \mathcal{Z}_{\Phi i} \\
-\sum_{a=1}^{n} \mathcal{Z}_{\Phi a}\left\{\frac{\partial^{2} V}{\partial \Phi_{i} \partial \Phi_{a}}+\frac{2}{3 \sqrt{-g}} \partial_{z}\left[\sqrt{-g} g^{z z} \Phi_{a}^{\prime} k_{i}\right]\right\}=0 .
\end{gathered}
$$

There are $n$ such equations, one for each of the $\mathcal{Z}_{\Phi i}$ variables. This system of $n$ coupled equations must be solved in order to determine the bulk spectral density in a generic multi-scalar gravity dual. (Usually, the complexity of the equations requires a numerical solution). If one is only interested in the bulk viscosity, one may set $w=0$ in the above equation.

The equation (43) comes from 20. It is clear from the derivation in that paper, that no special coordinate system is assumed other than the usual black brane ansatz:

$$
d s^{2}=g_{t t}(z) d t^{2}+g_{x x}(z) d \vec{x}^{2}+g_{z z}(z) d z^{2} .
$$

This justifies our discussion in the previous section; equation (43) is valid in any coordinate system with these symmetries, and so of course the set of solutions ( $R_{i}$ functions) is also independent of the coordinate system.

Let us now summarize the procedure to determine the bulk spectral density in this general multi-scalar model.

1. For the potential $V\left(\Phi_{1} \ldots \Phi_{n}\right)$ under consideration, one must solve the background equations of motion to determine $g_{x x}, g_{t t}, g_{z z}, \Phi_{i} \ldots \Phi_{n}$. Once these are known the $k_{i}$ functions are known. The relevant background equations are written in Appendix A

2. Once the background is determined, one must solve the set of equations (43) subject to the standard incoming wave condition. This is usually done by inserting the incoming wave ansatz

$$
\mathcal{Z}_{\Phi i}(z)=f(z)^{-i w / 4 \pi T} Y_{i}(z)
$$

with the assumption that $Y_{i}$ is regular at the horizon. A convenient way to do this numerically is to begin numerical integration at the horizon and integrate toward the boundary to determine the values of $\mathcal{Z}_{\Phi i}^{0}$. We refer the reader to [41] as the numerical procedure outlined there could be implemented here as well.

3. Once the boundary values $\mathcal{Z}_{\Phi i}^{0}$ are determined numerically, the $R_{i}$ functions are known, and hence one can use the formula (38) to determine the spectral density.

This completes our generalization of the work of [14 to multiple scalar fields. 


\section{B. Single scalar theories}

If only one scalar field is present, it is possible to completely remove the potential from the gauge invariant equations, since we can trade derivatives with respect to $\Phi$ for derivatives with respect to $z$ using the chain rule. After doing so and making judicious use of the background equations of motion (for details, see Appendix C), we find the single gauge invariant equation reduces to the following form. In the case of one scalar field, there is only one gauge invariant variable, and so we have dropped all the $i$ subscripts for simplicity:

$$
\begin{aligned}
& \frac{1}{\sqrt{-g}} \partial_{z}\left[\sqrt{-g} g^{z z} \mathcal{Z}_{\Phi}^{\prime}\right] \\
- & \mathcal{Z}_{\Phi}\left\{\frac{1}{\sqrt{-g} f k} \partial_{z}\left[\sqrt{-g} g^{z z} f k^{\prime}\right]+g^{t t} w^{2}\right\}=0 .
\end{aligned}
$$

The associated spectral density is

$$
\rho^{\mathrm{bulk}}(w)=\frac{s w}{\pi} \lim _{\epsilon \rightarrow 0} k(\epsilon)^{2} R(w, \epsilon) .
$$

\section{CHAMBLIN-REALL BACKGROUND}

Despite the relative simplicity of (46), it does not seem to be possible to solve the equation analytically in general, even in the limit of vanishing $w$. However, we are aware of one special case where certain analytical results are possible. This case is referred to as the ChamblinReall background, where the scalar potential is a pure exponential. The potential is defined as

$$
V(\Phi)=-\frac{6}{L^{2}} \frac{(2-\delta)}{(1-2 \delta)^{2}} \exp \left\{\sqrt{\frac{4 \delta}{3}} \Phi\right\}
$$

with the numerical factors chosen for later convenience. The parameter $L$ has dimensions of length; in the conformal case of $\delta=0$ it is the AdS curvature radius. The metric and background field are written as follows.

$$
\begin{aligned}
d s^{2} & =b^{2}(z)\left[-f(z) d t^{2}+d \mathbf{x}^{2}+\frac{d z^{2}}{f(z)}\right], \\
b(z) & =\left(\frac{L}{z}\right)^{1 /(1-2 \delta)}, \\
f(z) & =1-\left(\frac{z}{z_{h}}\right)^{2(2-\delta) /(1-2 \delta)}, \\
\Phi(z) & =-\sqrt{12 \delta} \log [b(z)] .
\end{aligned}
$$

The conformal symmetry breaking parameter $\delta$ ranges from $0 \leq \delta \leq 1 / 2$. It is related to the trace anomaly and speed of sound as [20, 37,

$$
\delta=\frac{\varepsilon-3 P}{2 \varepsilon}=\frac{1-3 v_{s}^{2}}{2}
$$

where $\varepsilon, P$, and $v_{s}$ denote the energy density, pressure, and speed of sound of the dual fluid. Note that the Chamblin-Reall background is special, because the function $k(z)$ is independent of $z$.

\section{A. Bulk spectral density and Euclidean correlators}

In this background, the equation of motion for $\mathcal{Z}_{\Phi}$ becomes

$$
\frac{1}{\sqrt{-g} g^{z z}} \partial_{z}\left[\sqrt{-g} g^{z z} \mathcal{Z}_{\Phi}^{\prime}\right]-\mathcal{Z}_{\Phi} g_{z z} g^{t t} w^{2}=0
$$

At this point, we notice an interesting coincidence between this equation for the bulk perturbation, and the case of the shear perturbation. In [41], the shear spectral density was examined in this model. In complete analogy with the expressions above, it was found that

$$
\rho^{\text {shear }}(w)=\frac{s w}{4 \pi}\left[\frac{H\left(w, z_{h}\right) H\left(-w, z_{h}\right)}{H(-w, \epsilon) H(w, \epsilon)}\right],
$$

with $H$ being the solution to the equation

$$
\frac{1}{\sqrt{-g} g^{z z}} \partial_{z}\left[\sqrt{-g} g^{z z} H^{\prime}\right]-w^{2} g_{z z} g^{t t} H=0 .
$$

It is clear that the equations for $H$ and for $\mathcal{Z}_{\Phi}$ are identical in this background only. (This fact was also recently pointed out in [49]). Using the fact that $H=\mathcal{Z}_{\Phi}$, we find that the bulk and shear spectral densities are very simply related; their ratio is a constant:

$$
\frac{\rho^{\text {bulk }}(w)}{\rho^{\text {shear }}(w)}=4 \lim _{z \rightarrow 0} k(z)^{2}=12 \delta .
$$

Applying the Kubo formulas to the case at hand, we see that

$$
\frac{\zeta}{\eta}=\frac{4}{3} \delta=2\left(\frac{1}{3}-v_{s}^{2}\right)
$$

which is a well known result (for example, see [14, 20]). One of the novel observations of this work is that the above ratio between $\zeta$ and $\eta$ is actually a special case of the more general fact that the full shear and bulk spectral densities are simply related at all values of $w$. In Appendix D, we re-derive this result using the methods of [14] and show that both methods give the same answer.

The shear spectral densities xwere computed in 41], and hence we can use the results given there to compute the bulk spectral densities. Some results are plotted in Fig. 1.

The spectral density is related to the full, Euclidean correlation function through an integral transform,

$$
G_{E}(\tau)=\frac{1}{\pi} \int d w \rho(w) \frac{\cosh [w(\tau-\beta / 2)]}{\sinh [w \beta / 2]},
$$

where $\tau$ is the Euclidean time variable, which has period $\beta \equiv 1 / T$. Euclidean correlation functions can be computed on the lattice. In Fig. 2 we plot this quantity (with the zero temperature part subtracted) in the bulk channel for the Chamblin-Reall background. Despite the oscillations in the spectral function, the Euclidean correlation functions turn out to be smooth. 
Zero-temperature subtracted bulk spectral density

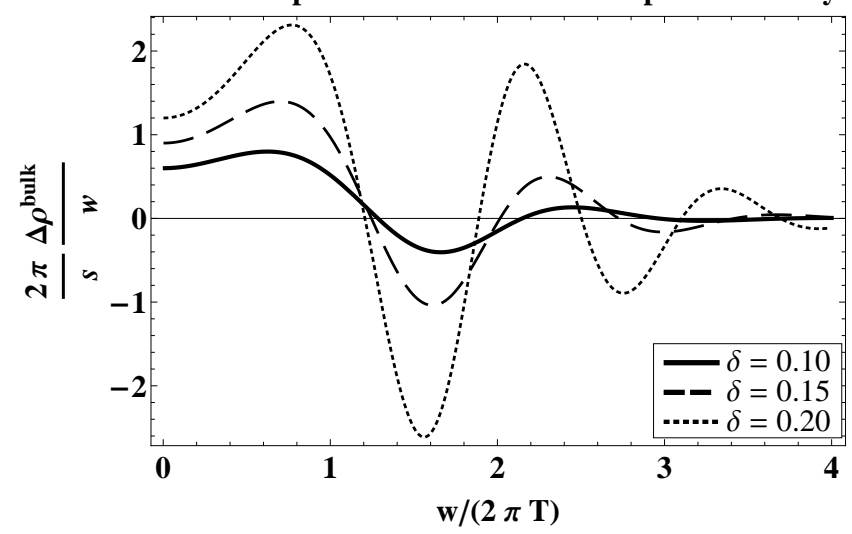

FIG. 1. Plots of the zero-temperature subtracted spectral density versus frequency for several values of $\delta$. For large $w$, the spectral density always approaches the zero-temperature result. Note that unlike the shear spectral density, the intercept at $w=0$ increases with $\delta$. This is a demonstration of the fact that the bulk viscosity increases with $\delta$. In a conformal theory, $(\delta=0)$, the spectral density vanishes identically.

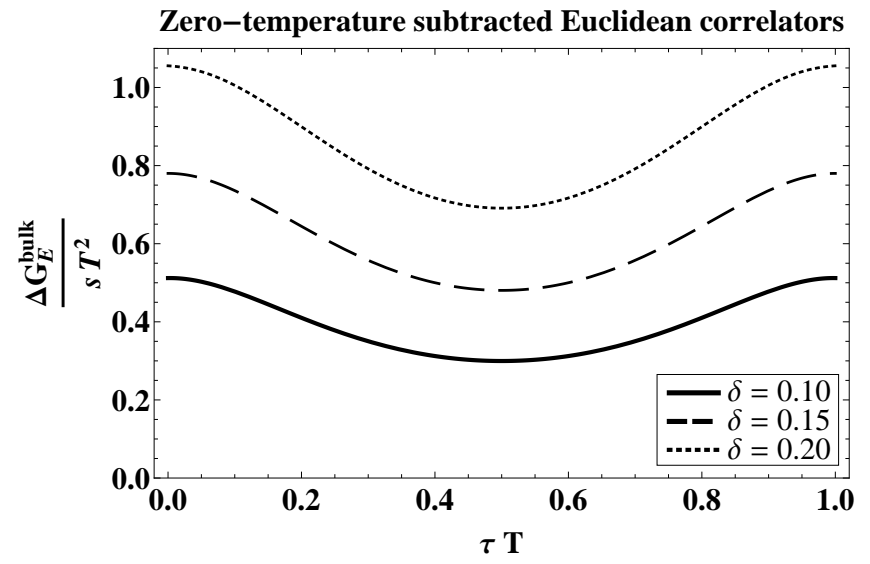

FIG. 2. Plot of the zero-temperature subtracted Euclidean correlation function associated with the bulk spectral density as a function of the Euclidean time $\tau$ for various values of $\delta$. In this model the value of $\delta$ (and hence the value of the bulk viscosity in this model) has a strong effect on the shape and magnitude of these functions. In a conformal theory, $(\delta=0)$, this function is identically zero.

\section{B. Bulk sum rule}

With the spectral density in hand, it is a simple matter to investigate the bulk sum rule of [40]. The shear sum rule in this background was already studied in [41, with the result

$$
\frac{3}{10-8 \delta}(\varepsilon+P)=\int_{0}^{\infty} \frac{d w}{w}\left[\rho^{\text {shear }}(w)-\rho_{\mathrm{T}=0}^{\text {shear }}(w)\right] .
$$

Multiplying both sides by $12 \delta$, and using (57), we find the result for the bulk channel

$$
\frac{36 \delta}{10-8 \delta}(\varepsilon+P)=\int_{0}^{\infty} \frac{d w}{w}\left[\rho^{\text {bulk }}(w)-\rho_{\mathrm{T}=0}^{\mathrm{bulk}}(w)\right] .
$$

In [40, the left side of the above sum rule was derived for Yang-Mills theories, and was found to take on the value

$$
3(\varepsilon+P)\left(1-3 v_{s}^{2}\right)-4(\varepsilon-3 P) .
$$

If we naively evaluate this quantity in the Chamblin-Reall metric using (53), we find

$$
3(\varepsilon+P)\left(1-3 v_{s}^{2}\right)-4(\varepsilon-3 P)=\frac{-6 \delta^{2}}{2-\delta}(\varepsilon+P) .
$$

Clearly, there are differences between the bulk sum rule of 40 and the bulk sum rule for the Chamblin-Reall metric (both the sign and the leading power of $\delta$ are different).

What is the nature of this difference? The derivation of the sum rule 40. employs asymptotic freedom, and so we should examine the beta function in the ChamblinReall background to see whether this model meets the necessary requirements.

\section{Beta function and trace anomaly}

One can show that in single scalar gravity duals, the trace anomaly is consistent with that of QCD,

$$
\left\langle\operatorname{Tr}\left(F^{2}\right)\right\rangle=4(\varepsilon-3 P)\left(\frac{\lambda_{t}^{2}}{\beta\left(\lambda_{t}\right)}\right),
$$

provided that we identify 18

$$
\begin{aligned}
\left\langle\operatorname{Tr}\left(F^{2}\right)\right\rangle & =\lambda_{t} \frac{8 \sqrt{2}}{\sqrt{3}}\left\langle\mathcal{O}_{\Phi}\right\rangle, \\
\frac{\beta\left(\lambda_{t}\right)}{\lambda_{t}} & =\sqrt{\frac{3}{8}} b \frac{d \Phi}{d b} .
\end{aligned}
$$

Here $\lambda_{t}=g^{2} N_{c}$ is the t'Hooft coupling, $F^{2}$ is the square of the field strength tensor $F_{\mu \nu}^{a}$, and $b$ is defined from the metric as written in the coordinate system (49). The field theory operator dual to the scalar field $\Phi$ is denoted as $\mathcal{O}_{\Phi}$. The t'Hooft coupling is usually identified with a scaled version of the dilaton

$$
e^{\sqrt{\frac{3}{8}} \Phi}=c_{0} \lambda_{t}
$$

with $c_{0}$ being an unknown proportionality constant which will not enter our equations. Note that

$$
\frac{\beta\left(\lambda_{t}\right)}{\lambda_{t}}=\sqrt{\frac{3}{8}} b(z) \frac{d \Phi}{d z} \frac{d z}{d b}=\sqrt{\frac{3}{2}} \frac{\Phi^{\prime}(z) g_{x x}(z)}{g_{x x}^{\prime}(z)}=\sqrt{\frac{3}{2}} k(z) .
$$

In order to get an explicit result for $\beta$ in the general case, one would have to invert $\lambda_{t}(z)$ and substitute it above to get $k\left(z\left(\lambda_{t}\right)\right)$. In the case of the Chamblin-Reall metric, 
this is unnecessary because $k$ is a pure constant. In this background, then

$$
\beta\left(\lambda_{t}\right)=-3 \lambda_{t} \sqrt{\frac{\delta}{2}}
$$

The beta function is negative, and hence this model possesses asymptotic freedom. There must be another reason why the sum rule in this model does not agree with that of 40 .

The left side of the sum rule is, more generally,

$$
\Delta G_{R}^{\mathrm{bulk}}(w=i \infty)-\Delta G_{R}^{\mathrm{bulk}}(w=0),
$$

where we use the symbol $\Delta$ to denote a zero-temperature subtraction as in (7). The low energy piece (at $w=0$ ) provides a universal contribution from hydrodynamics, so this is not the source of the difference. The crucial difference is the source of conformal symmetry breaking in each model. In gauge theories, the conformal symmetry is broken by the running of the coupling. If the theory is asymptotically free, at large energy scales the coupling vanishes due to asymptotic freedom, and hence the high energy term (at $w=i \infty)$ vanishes. In other words, conformal symmetry is restored at high energies or temperatures in such gauge theories.

In the Chamblin-Reall theory, there is a hard breaking of conformal symmetry due to a parameter in the Lagrangian. This parameter does not run with energy scale and hence at large energies or temperatures, conformal symmetry is not restored, and thus $\Delta G_{R}(w=i \infty)$ does not vanish in general. Thus, the different form of the left side of the sum rule in this theory is a consequence of the fact that the conformal symmetry breaking is present for all temperatures.

Despite the fact that the left side of the sum rule appears different in each theory, we can find some similarity by writing the left side in terms of the beta function. In what follows, we will assume that the conformal symmetry breaking is small $(\varepsilon-3 P) \ll \varepsilon, P$.

\section{Yang-Mills theory}

For gauge theories, the region of small conformal symmetry breaking occurs at weak coupling. The pressure can be computed as

$$
P(T)=T^{4}\left(A+B g^{2}+\mathcal{O}\left(g^{3}\right)\right) .
$$

using $s=P^{\prime}(T), \epsilon+P=T s$ and $v_{s}^{2}=P^{\prime}(T) / \varepsilon^{\prime}(T)$, one finds that, to lowest order in the coupling constant $g$, the left hand side of the Romastschke-Son sum rule is

$$
3(\varepsilon+P)\left(1-3 v_{s}^{2}\right)-4(\varepsilon-3 P) \approx B T^{5} \frac{d \beta\left(g^{2}\right)}{d T},
$$

Where

$$
\beta\left(g^{2}\right)=T \frac{d g^{2}}{d T}
$$

The beta function is computed as

$$
\beta\left(g^{2}\right)=-b_{0} g^{4}+b_{1} g^{6}+\ldots
$$

Again, to leading order,

$$
T \frac{d \beta\left(g^{2}\right)}{d T}=-2 b_{0} g^{2} \beta\left(g^{2}\right)=2\left(\frac{\beta\left(g^{2}\right)}{g}\right)^{2} .
$$

In all, then the left side of the sum rule becomes (to leading order in the conformal symmetry breaking parameter, $g)$,

$$
3(\varepsilon+P)\left(1-3 v_{s}^{2}\right)-4(\varepsilon-3 P) \approx \frac{2 B}{A} P_{0}\left(\frac{\beta\left(g^{2}\right)}{g}\right)^{2}
$$

where $P_{0}$ denotes the pressure in the conformal limit $P_{0}=A T^{4}$. Inserting factors of $N_{c}$ and using the fact that the t'Hooft coupling is $\lambda_{t}=g^{2} N_{c}$, we find

$$
3(\varepsilon+P)\left(1-3 v_{s}^{2}\right)-4(\varepsilon-3 P) \approx \frac{2 B \lambda_{t}}{A N_{c}} P_{0}\left(\frac{\beta\left(\lambda_{t}\right)}{\lambda_{t}}\right)^{2} .
$$

\section{Chamblin-Reall background}

For the Chamblin-Reall background, the conformal symmetry breaking parameter is $\delta$. The left side of the sum rule is to lowest order:

$$
\frac{18}{5} \delta \times 4 P_{0}+\mathcal{O}\left(\delta^{2}\right)
$$

Here, again, $P_{0}$ denotes the pressure in the conformal limit $P_{0}=P(\delta \rightarrow 0)$, and we have used the fact that $\varepsilon=3 P$ in the conformal limit. Employing the use of the beta function

$$
\left(\frac{\beta\left(\lambda_{t}\right)}{\lambda_{t}}\right)^{2}=\frac{9}{2} \delta
$$

we find the left side of the sum rule can be written

$$
\frac{16}{5} P_{0}\left(\frac{\beta\left(\lambda_{t}\right)}{\lambda_{t}}\right)^{2}
$$

Comparing (80) to (77), we note that when the conformal symmetry breaking is small, the bulk sum rule is well approximated in both theories as

$$
C P_{0}\left(\frac{\beta\left(\lambda_{t}\right)}{\lambda_{t}}\right)^{2} \approx \int_{0}^{\infty} \frac{d w}{w}\left[\rho^{\text {bulk }}(w)-\rho_{\mathrm{T}=0}^{\text {bulk }}(w)\right]
$$

This equation is approximate because we have only included the leading order term in the deviation from conformality on the left side. While the functional form is similar in both theories, $C$ is theory dependent. For the Chamblin-Reall background we have shown $C=16 / 5$; for pure Yang-Mills theory [50], it is $C=-5 \lambda_{t} / 8 \pi^{2}$. It would be interesting to see whether this dependence 
holds in other theories beyond those considered here. It is interesting that $C$ contains a different dependence on the coupling in each theory; this is a reflection of the fact that to lowest order the beta function goes as $\lambda_{t}$ in Chamblin-Reall, but as $\lambda_{t}^{2}$ in Yang-Mills theory.

In the theories we have considered, the left hand side of the bulk sum rule has the same dependence (on the conformal symmetry breaking parameter) as the bulk viscosity. In fact, it has been argued on general grounds that the bulk viscosity should be proportional to two factors of the beta function in 51. Despite the fact that the sum rule (and bulk viscosity) both depend on two powers of the beta function in both theories, the dependence on $(\varepsilon-3 P)$ is different. By looking at the Kubo formula for the bulk viscosity one might expect the dependence $\zeta \sim(\varepsilon-3 P)^{2}$. Here, we see this is not generally true. The quantity $\varepsilon-3 P$ is not necessarily linearly proportional to the beta function.

\section{CONCLUSION}

The main results of this work were presented in two parts. First, we presented a prescription for calculating the bulk spectral density (and hence the bulk viscosity) for a general, five-dimensional multi-scalar gravity dual theory. In this way, we have generalized the results of [14] to multiple scalar fields. In so doing, we have calculated the on-shell gravitational action relevant for the computation of bulk mode two point correlation functions. We have also given explicitly the set of gauge invariant equations which need to be solved for a given background. As such, our main results for this part are summarized at the end of Sec. IV

There are many gravitational dual theories on the market today. For this reason, it would be useful to have a definite prescription which allows for the calculation of the bulk spectral function which works for a large class of gravity duals. We have taken a step in this direction by including multiple scalar fields. Also, as mentioned in the introduction, more than one scalar field often appears when deriving a five-dimensional effective action for a string theory setup. Hence, the methods we have developed here could aid future computations in backgrounds like those of [27, 28].

In the second part of the paper, we used the methods derived in the first part to compute the bulk spectral function (at vanishing spatial momentum $\vec{q}$ ) in the Chamblin-Reall background. We noticed the intriguing fact that in this model the ratio of the bulk to shear spectral densities is a pure constant. We also derived a sum rule for the bulk channel and show that it is different from the sum rule of Romatschke and Son [40. The reason for this difference is not due to asymptotic freedom, but rather due to the "hard" nature of the conformal symmetry breaking in the Chamblin-Reall background. We have also shown that (to leading order in the conformal symmetry breaking) the left side of the sum rule in both Yang-Mills theory and in the Chamblin-Reall background is proportional to two factors of the beta function.

When one attempts to extract transport coefficients from the lattice, one typically needs to make an ansatz for the spectral function. The results from AdS/CFT computations like the one presented here could be used in this process (though, as we mentioned previously, it is necessary to perform the computation in a theory which shares more phenomenological features with QCD).

There are many possible directions for future work. It would be useful to further generalize our prescription by including other matter fields beyond the simple scalars included here. The inclusion of vector fields allows one to study holographic models at nonzero chemical potential. One could also try to generalize our methods to an arbitrary number of dimensions. Furthermore, it would be interesting to compute the correlation functions at nonvanishing spatial momentum. In this way, one could have a complete generalization of the known conformal results of [52]. We hope to address some of these issues in future publications.

\section{ACKNOWLEDGMENTS}

We thank Aldo Cotrone, Paul Romatschke, Dam Son and Mikhail Stephanov for helpful discussions. This work was supported by the Natural Sciences and Engineering Research Council of Canada.

\section{Appendix A: Equations of motion}

In this section we write down the relevant background Einstein equations and the linearized Einstein equations for the perturbations.

\section{Background equations}

There are $3+n$ independent background equations, corresponding to three metric components $g_{t t}, g_{x x}, g_{z z}$ and $n$ scalar fields $\Phi_{i}$. For later convenience, we write these equations as the vanishing of the quantities

$$
\mathcal{E}_{1}^{(0)}=\mathcal{E}_{2}^{(0)}=\mathcal{E}_{3}^{(0)}=\mathcal{E}_{\Phi i}^{(0)}=0
$$

with the definitions

$$
\begin{aligned}
& \mathcal{E}_{1}^{(0)} \equiv V\left(\Phi_{1}, \ldots \Phi_{n}\right)+\frac{3}{2 \sqrt{-g}} \partial_{z}\left(\sqrt{-g} g^{z z} \mathcal{D}_{L}\left[g_{x x}\right]\right)(\mathrm{A} 2) \\
& \mathcal{E}_{2}^{(0)} \equiv \Phi_{a}^{\prime} \Phi_{a}^{\prime}-\frac{3}{2} \mathcal{D}_{L}\left[g_{x x}\right] \mathcal{D}_{L}\left[\frac{g_{z z} f}{\mathcal{D}_{L}\left[g_{x x}\right]^{2}}\right] \\
& \mathcal{E}_{3}^{(0)} \equiv \frac{1}{2 \sqrt{-g}} \partial_{z}\left(\sqrt{-g} g^{z z} \mathcal{D}_{L}[f]\right) \\
& \mathcal{E}_{\Phi i}^{(0)}=\frac{\partial V}{\partial \Phi_{i}}-\frac{1}{\sqrt{-g}} \partial_{z}\left(\sqrt{-g} g^{z z} \Phi_{i}^{\prime}\right) .
\end{aligned}
$$


The superscript (0) denotes that these are background (zeroth order) equations. The logarithmic derivative $\mathcal{D}_{L}$ was introduced in (41). A summation over the repeated index $a$ is implied.

\section{Linearized equations}

There are $2+\mathrm{n}$ independent linearized Einstein equations (first order in the perturbations) corresponding to the two metric perturbations $A, B$ and the $n$ scalar perturbations $\varphi_{i}$. In [20], the linearized perturbations for this system were given in the more general case of $\vec{q} \neq 0$; in the general case there are 4 metric perturbations denoted as $A, B, C, D$. One can get the equations necessary for the current work by examining the equations in [20] in the limit of $q=0, D=0, B=C$. We will write the equations for the Fourier transformed perturbations, such as

$$
A(t, z)=\int \frac{d w}{2 \pi} A(w, z) e^{-i w t}
$$

with the shorthand $A_{w}, B_{w}, \varphi_{i, w}$ denoting $A(w, z)$, $B(w, z), \varphi_{i}(w, z)$. As in the previous subsection we write these equations as the vanishing of the quantities

$$
\mathcal{E}_{1}^{(1)}=\mathcal{E}_{2}^{(1)}=\mathcal{E}_{\Phi i}^{(1)}=0
$$

with the definitions

$$
\begin{gathered}
\mathcal{E}_{1}^{(1)} \equiv 3 \sqrt{f} \partial_{z}\left[\frac{B_{w}}{\sqrt{f}}\right]+\Phi_{a}^{\prime} \varphi_{a, w} \\
\mathcal{E}_{2}^{(1)} \equiv \mathcal{D}_{L}\left[g_{x x}^{3}\right] A_{w}^{\prime}+3 \mathcal{D}_{L}\left[g_{x x}^{2} g_{t t}\right] B_{w}^{\prime}-2 \Phi_{a}^{\prime} \varphi_{a, w}^{\prime} \\
+\frac{2 \varphi_{a, w}}{\sqrt{-g} g^{z z}} \partial_{z}\left[\sqrt{-g} g^{z z} \Phi_{a}^{\prime}\right]-6 w^{2} g_{z z} g^{t t} B_{w},(\mathrm{~A}) \\
\mathcal{E}_{\Phi i}^{(1)} \equiv \frac{1}{\sqrt{-g} g^{z z}} \partial_{z}\left[\sqrt{-g} g^{z z} \varphi_{i, w}^{\prime}\right]+\frac{\Phi_{i}^{\prime}}{2}\left(A_{w}^{\prime}+3 B_{w}^{\prime}\right) \\
-g_{z z} \frac{\partial^{2} V}{\partial \Phi_{i} \partial \Phi_{a}} \varphi_{a, w}-w^{2} g_{z z} g^{t t} \varphi_{i, w} .
\end{gathered}
$$

Summation over the repeated index $a$ is implied, and we have used the background equations to remove any reference to the potential in A9

\section{Appendix B: Derivation of on-shell action}

In this section, we provide a calculation of the on-shell action in terms of gauge invariant variables, leading to the expressions (22) and (24). Throughout this section we will assume that the metric is written in the coordinate system (21). Because it is sometimes cumbersome to write down square matrices of arbitrary rank, we will write any necessary matrices for two scalar fields. It will be immediately obvious how to generalize our final result for more than two scalar fields.

To begin, we start with the action (8) and the perturbations defined in $(14,15)$. Next, one expands the action to second order in the perturbations.

\section{Bulk term}

We first focus on the "bulk term": the first term in (8). The part quadratic in the perturbations can be written

$$
\begin{aligned}
\mathcal{S}_{2}^{\text {bulk }} & =\frac{1}{2 \kappa} \int d^{5} x\left[\mathcal{Q}^{T} M \mathcal{Q}+\left(\mathcal{Q}^{\prime}\right)^{T} M_{z z} \mathcal{Q}^{\prime}+\left(\mathcal{Q}^{\prime}\right)^{T} M_{z} \mathcal{Q}\right. \\
& \left.+\left(\mathcal{Q}^{\prime \prime}\right)^{T} M_{2 z} \mathcal{Q}+(\ddot{\mathcal{Q}})^{T} M_{2 t} \mathcal{Q}+(\dot{\mathcal{Q}})^{T} M_{t t} \dot{\mathcal{Q}}\right] . \quad(\mathrm{B} 1)
\end{aligned}
$$

Here, $\mathcal{Q}$ is the matrix of perturbations

$$
\mathcal{Q}(t, z)=\left(\begin{array}{c}
A(t, z) \\
B(t, z) \\
\varphi_{1}(t, z) \\
\vdots \\
\varphi_{n}(t, z)
\end{array}\right)
$$

the dot denotes derivatives with respect to time, and the prime denotes derivatives with respect to $z$. And we have introduced several $2+n$ by $2+n$ matrices: $M, M_{z z}$, etc. We will not write down the components of all these matrices explicitly here, as only a fraction of these components enter into our final result.

We will work with the Fourier modes, by inserting

$$
\mathcal{Q}(t, z)=\int \frac{d w}{2 \pi} Q(w, z) e^{-i w t} .
$$

And we will employ the shorthand

$$
Q_{w}=Q(w, z)
$$

The on-shell action becomes

$$
\begin{aligned}
\mathcal{S}_{2}^{\text {bulk }} & =\frac{1}{4 \kappa} \int d^{3} x d z \frac{d w}{2 \pi}\left\{2\left(Q_{w}^{\prime}\right)^{T} M_{z z} Q_{-w}^{\prime}\right. \\
& +\left(Q_{w}^{\prime}\right)^{T} M_{z} Q_{-w}+Q_{w}^{T} M_{z}^{T} Q_{-w}^{\prime} \\
& \left.+\left(Q_{w}^{\prime \prime}\right)^{T} M_{2 z} Q_{-w}+Q_{w}^{T} M_{2 z}^{T} Q_{-w}^{\prime \prime}+Q_{w}^{T} M_{c} Q_{-w}\right\}
\end{aligned}
$$

with $M_{c}$ defined as

$$
M_{c} \equiv 2 M+w^{2}\left(M_{t t}+M_{t t}^{T}-M_{2 t}-M_{2 t}^{T}\right) .
$$

Note that because of the integration over all $w$, (from $-\infty$ to $\infty$ ) we have taken the opportunity to symmetrize the entire expression in $w \rightarrow-w$. Terms which are odd in $w$ will drop out under the $w$ integration. Integrating 
by parts to get an action which depends only on first derivatives, one has

$$
\begin{aligned}
\mathcal{S}_{2}^{\text {bulk }} & =\frac{V_{3}}{4 \kappa} \int d z \frac{d w}{2 \pi}\left[\left(Q_{w}^{\prime}\right)^{T} M_{a} Q_{-w}^{\prime}+\left(Q_{w}^{\prime}\right)^{T} M_{b} Q_{-w}\right. \\
& \left.+Q_{w}^{T} M_{b}^{T} Q_{-w}^{\prime}+Q_{w}^{T} M_{c} Q_{-w}+\partial_{z} \Delta(w, z)\right] \quad(\mathrm{B} 6)
\end{aligned}
$$

with

$$
\begin{aligned}
M_{a}(z) & \equiv 2 M_{z z}-M_{2 z}-M_{2 z}^{T} \\
M_{b}(z) & \equiv M_{z}-M_{2 z}^{\prime} \\
\Delta(w, z) & \equiv\left(Q_{w}^{\prime}\right)^{T} M_{2 z} Q_{-w}+Q_{w}^{T} M_{2 z}^{T} Q_{-w},
\end{aligned}
$$

and we have introduced the result of the integration over $d^{3} x$ as $V_{3}$. One can now employ the Euler-Lagrange equations to get the equations of motion for the perturbations. In doing so, one should treat $Q_{w}$ and $Q_{-w}$ as separate functions. (The reason is that one has in mind a complex perturbation so that $Q_{-w} \sim Q_{w}^{*}$ 14.) One can show that upon application of the equations of motion, this entire term in the on-shell action reduces to a total derivative term. This procedure has been explained many times in the literature (e.g. [14, 48, 53]) hence we will not go through all of the details. The result of this procedure is

$$
\begin{aligned}
\mathcal{S}_{2}^{\text {bulk }} & =\frac{V_{3}}{4 \kappa} \int \frac{d w}{2 \pi}\left[\left(Q_{w}^{\prime}\right)^{T}\left(M_{a}+M_{2 z}\right) Q_{-w}\right. \\
& \left.+Q_{w}^{T} M_{2 z}^{T} Q_{-w}^{\prime}+Q_{w}^{T} M_{b}^{T} Q_{-w}\right]_{z=z_{B}} .
\end{aligned}
$$

Here $z=z_{B}$ is a symbolic way of noting that the above should be evaluated at the boundary of the space-time.

\section{Gibbons-Hawking term}

We now must add the second term of (8), which is itself a pure boundary term. After expanding this term to second order in the perturbations, we find

$$
\mathcal{S}_{2}^{G H}=\frac{1}{2 \kappa} \int\left(\mathcal{Q}^{\prime}\right)^{T} M_{G H a} \mathcal{Q}+\mathcal{Q}^{T} M_{G H b} \mathcal{Q} .
$$

with two new matrices $M_{G H a}, M_{G H b}$. Repeating the arguments of the previous section, by inserting the Fourier modes and symmetrizing in $w$, we find the GibbonsHawking contribution

$$
\begin{aligned}
\mathcal{S}_{2}^{G H} & =\frac{V_{3}}{4 \kappa} \int \frac{d w}{2 \pi}\left[\left(Q_{w}^{\prime}\right)^{T} M_{G H a} Q_{-w}+Q_{w}^{T} M_{G H a}^{T} Q_{-w}^{\prime}\right. \\
& \left.+Q_{w}^{T}\left(M_{G H b}+M_{G H b}^{T}\right) Q_{-w}\right]_{z=z_{B}} .
\end{aligned}
$$

By an explicit computation, we find that both $M_{2 z}$ and $M_{G H a}$ are symmetric, and that

$$
M_{G H a}=-M_{2 z} .
$$

Indeed, this should come as no surprise, since the purpose of the Gibbons-Hawking term is to cancel off the boundary contribution due to the integration by parts.

\section{Total}

Adding together the bulk and Gibbons-Hawking terms, and using (B13), we find

$$
\begin{aligned}
\mathcal{S}_{2} & =\mathcal{S}_{2}^{\text {bulk }}+\mathcal{S}_{2}^{G H} \\
& =\frac{V_{3}}{4 \kappa} \int \frac{d w}{2 \pi}\left(Q_{w}^{\prime}\right)^{T} M_{a} Q_{-w}+Q_{w}^{T}\left(M_{b}^{T}+2 M_{G H b}\right) Q_{-w} .
\end{aligned}
$$

We are now ready to write down the forms of the matrices appearing here explicitly. For the case of two scalar fields, the matrices are $4 \times 4$, and are given by

$$
M_{a}=\frac{f g_{x x}^{3 / 2}}{2}\left(\begin{array}{cccc}
0 & 3 & 0 & 0 \\
3 & 6 & 0 & 0 \\
0 & 0 & -2 & 0 \\
0 & 0 & 0 & -2
\end{array}\right)
$$

and

$$
\begin{aligned}
& M_{b}^{T}+2 M_{G H b}= \\
& \frac{f g_{x x}^{3 / 2}}{4}\left(\begin{array}{cccc}
-3 \mathcal{D}_{L}\left[g_{x x}\right] & 3 \mathcal{D}_{L}\left[f g_{x x}^{3}\right] & -2 \Phi_{1}^{\prime} & -2 \Phi_{2}^{\prime} \\
9 \mathcal{D}_{L}\left[g_{x x}\right] & 3 \mathcal{D}_{L}\left[f g_{x x}^{3}\right] & -6 \Phi_{1}^{\prime} & -6 \Phi_{2}^{\prime} \\
0 & 0 & 0 & 0 \\
0 & 0 & 0 & 0
\end{array}\right)
\end{aligned}
$$

It should be evident how to generalize these matrices for more than two scalars. Regardless of the number of scalars, the $2 \times 2$ submatrix in the upper left corner always remains the same, since these entries correspond to the metric perturbations $A$ and $B$. For more scalar fields, $M_{a}$ simply has more entries of -2 along the diagonal. Similarly, (B16) will in general only have non-zero entries in the first two rows; one adds entries $-2 \Phi_{3}^{\prime} \ldots-2 \Phi_{n}^{\prime}$ to the first row, and $-6 \Phi_{3}^{\prime} \ldots-6 \Phi_{n}^{\prime}$ to the second row.

\section{Gauge invariant form}

We would like to write the action involving the gaugeinvariant variables $\mathcal{Z}_{\Phi i}$. Our strategy is to employ the equations of motion to remove all derivatives except for $\mathcal{Z}_{\Phi i}^{\prime}$. Specifically, we first use A9 to remove all instances of $A^{\prime}$, then remove all $\varphi^{\prime}$ in favor of $\mathcal{Z}_{\Phi i}^{\prime}$ and $B^{\prime}$. Finally, use A8 to remove all remaining $B^{\prime}$ terms. The result is

$$
\begin{aligned}
& \mathcal{S}_{2}-\frac{V_{3}}{8 \kappa} \int \frac{d w}{2 \pi} \frac{g_{x x}^{5 / 2} f}{g_{x x}^{\prime}}\left\{B_{-w} \mathcal{E}_{2}^{(1)}-\frac{2 \mathcal{Z}_{\Phi a}(-w, z) \Phi_{a}^{\prime}}{3} \mathcal{E}_{1}^{(1)}\right. \\
- & {\left.\left[\mathcal{D}_{L}\left[g_{x x} f\right] B_{-w}-\mathcal{D}_{L}\left[g_{x x}\right] A_{-w}\right] \mathcal{E}_{1}^{(1)}\right\} } \\
= & \frac{-V_{3}}{4 \kappa} \int \frac{d w}{2 \pi} g_{x x}^{3 / 2} f\left\{\mathcal{Z}_{\Phi a}(-w, z) \mathcal{Z}_{\Phi a}^{\prime}(w, z)+Q_{w}^{T} \xi Q_{-w}\right\}
\end{aligned}
$$

The terms on the left side vanish on-shell due to the equations of motion $\mathcal{E}_{1}^{(1)}=\mathcal{E}_{2}^{(1)}=0$. The term without 
derivatives (the contact term) contains the matrix $\xi$, the components of which can be written (in the case of two scalar fields):

$$
\xi(z) \equiv\left(\begin{array}{cccc}
\frac{3}{4} \mathcal{D}_{L}\left[g_{x x}\right] & -\frac{3}{4} \mathcal{D}_{L}\left[f g_{x x}^{3}\right] & \frac{\Phi_{1}^{\prime}}{2} & \frac{\Phi_{2}^{\prime}}{2} \\
-\frac{3}{4} \mathcal{D}_{L}\left[f g_{x x}^{3}\right] & \frac{3}{4} \chi(z) & k_{1} \mathcal{D}_{L}\left[k_{1} g_{x x}^{3 / 2} \sqrt{f}\right] & k_{2} \mathcal{D}_{L}\left[k_{2} g_{x x}^{3 / 2} \sqrt{f}\right] \\
\frac{\Phi_{1}^{\prime}}{2} & k_{1} \mathcal{D}_{L}\left[k_{1} g_{x x}^{3 / 2} \sqrt{f}\right] & -\frac{\Phi_{1}^{2}}{3 \mathcal{D}_{L}} & -\frac{\Phi_{1}^{\prime} \Phi_{2}^{\prime}}{3 \mathcal{D}_{L}\left[g_{x x}\right]} \\
\frac{\Phi_{2}^{\prime}}{2} & k_{2} \mathcal{D}_{L}\left[k_{2} g_{x x}^{3 / 2} \sqrt{f]}\right. & -\frac{\Phi_{1}^{\prime} \Phi_{2}^{\prime}}{3 \mathcal{D}_{L}\left[g_{x x}\right]} & -\frac{\Phi_{1}^{2}}{3 \mathcal{D}_{L}\left[g_{x x}\right]}
\end{array}\right)-\left(\begin{array}{cccc}
0 & 0 & 0 & 0 \\
0 & \frac{1}{2 f} \partial_{z}\left(\frac{f \mathcal{E}_{2}^{(0)}}{\mathcal{D}_{L}\left[g_{x x}\right]^{2}}\right) & 0 & 0 \\
0 & -\frac{k_{1}}{3 \mathcal{D}_{L}\left[g_{x x}\right]} \mathcal{E}_{2}^{(0)} & 0 & 0 \\
0 & -\frac{k_{2}}{3 \mathcal{D}_{L}\left[g_{x x}\right]} \mathcal{E}_{2}^{(0)} & 0 & 0
\end{array}\right)
$$

The second matrix here is proportional to the background equations of motion, and thus vanishes on-shell. The function $\chi(z)$ is defined as

$$
\chi(z) \equiv \frac{\mathcal{D}_{L}[f]^{2}}{\mathcal{D}_{L}\left[g_{x x}\right]}-\mathcal{D}_{L}\left[f g_{x x}^{3}\right]-\frac{2}{f} \partial_{z}\left[f \partial_{z}\left(\frac{g_{x x}}{g_{x x}^{\prime}}\right)\right]+\frac{4 w^{2} g_{x x}}{f^{2} g_{x x}^{\prime}} .
$$

It is clear that $\xi$ is a symmetric matrix (up to terms which vanish upon application of the equations of motion). For this reason, it does not enter in the computation of the spectral density which we consider in this work. The matrix $\xi$ may be useful in future work, if one is interested in computing the real part of the correlation functions. Such a computation would also require extra counter terms to renormalize the on-shell action. In the present work, we do not need to worry about these complications, as these counter terms are real, and do not contribute to the spectral functions.

The equation (B17) is the final form for the action in terms of the gauge invariant variables. Taking the imaginary part of the action

$$
\operatorname{Im} \mathcal{S}_{2}(w)=\frac{1}{2 i}\left(\mathcal{S}_{2}(w)-\mathcal{S}_{2}(-w)\right),
$$

and using the relations for temperature and entropy

$$
\begin{aligned}
s & =\frac{2 \pi g_{x x}\left(z_{h}\right)^{3 / 2}}{\kappa} \\
T & =-\frac{f^{\prime}\left(z_{h}\right)}{4 \pi}
\end{aligned}
$$

we arrive at the form quoted in the text, Eq. (24).

\section{Appendix C: Derivation of the equation for $\mathcal{Z}_{\Phi}$ for a single scalar}

In this section we provide the detailed steps which allow one to derive 46 from (43) in the case of a single scalar field. Let us write equation (43) as

$$
\mathcal{E}_{Z}^{(1)}=0
$$

with

$$
\begin{aligned}
\mathcal{E}_{Z}^{(1)} & \equiv \frac{1}{\sqrt{-g}} \partial_{z}\left[\sqrt{-g} g^{z z} \mathcal{Z}_{\Phi}^{\prime}\right]-w^{2} g^{t t} \mathcal{Z}_{\Phi} \\
& -\mathcal{Z}_{\Phi}\left\{\frac{\partial^{2} V}{\partial \Phi^{2}}+\frac{2}{3 \sqrt{-g}} \partial_{z}\left[\sqrt{-g} g^{z z} \Phi^{\prime} k\right]\right\}
\end{aligned}
$$

We now add the following combination of the background equations A2 - A5 to get

$$
\begin{aligned}
\mathcal{E}_{Z}^{(1)} & +\frac{\mathcal{Z}_{\Phi}}{\mathcal{D}_{L}\left[g_{x x}\right]}\left\{\frac{1}{k} \partial_{z}\left(\mathcal{E}_{\Phi}^{(0)}\right)+2 \mathcal{D}_{L}\left[g_{x x}\right] \mathcal{E}_{3}^{(0)}\right. \\
& \left.+\frac{1}{3} \frac{g_{z z}}{(k \sqrt{-g})^{2}} \partial_{z}\left[\left(k \sqrt{-g} g^{z z}\right)^{2} \mathcal{E}_{2}^{(0)}\right]\right\}=0
\end{aligned}
$$

Carrying out the algebra, we find that this reduces to

$$
\begin{aligned}
& \frac{1}{\sqrt{-g}} \partial_{z}\left[\sqrt{-g} g^{z z} \mathcal{Z}_{\Phi}^{\prime}\right] \\
- & \mathcal{Z}_{\Phi}\left\{w^{2} g^{t t}+\frac{1}{f k \sqrt{-g}} \partial_{z}\left[\sqrt{-g} g^{z z} f k^{\prime}\right]\right\}=0
\end{aligned}
$$

which is the equation presented in the text 46 .

\section{Appendix D: Chamblin-Reall spectral function - comparison with Gubser et al.}

In this section, we compute the spectral density $\rho^{\text {bulk }}$ in the Chamblin-Reall model using the method of Gubser et al. 14. The results are consistent with those given in Sec. VA, though our methods are more generally applicable to theories with multiple scalar fields. The notations and conventions of this section are not the same as those given in the rest of the present work. Instead, the notation here is the same as that in [14. At times we have inserted some dimensionful factors for clarity.

The potential is written as

$$
V(\Phi)=V_{0} e^{\gamma \Phi}
$$

with $0 \leq \gamma \leq \sqrt{2 / 3}$. The background is written in coordinates where the scalar field is a scaled version of the radial coordinate (which we will call $r$ throughout this section):

$$
d s^{2}=e^{2 A(r)}\left[-h(r) d t^{2}+d \vec{x}^{2}\right]+e^{2 B(r)} \frac{d r^{2}}{h(r)}
$$




$$
\begin{aligned}
& A(r)=-\frac{\sqrt{-V_{0}}}{3 \gamma} r \\
& B(r)=\frac{1}{2}\left(\log \left[\frac{8-3 \gamma^{2}}{6 \gamma^{2}}\right]-r \gamma \sqrt{-V_{0}}\right) \\
& h(r)=1-\exp \left\{\frac{\sqrt{-V_{0}}\left(8-3 \gamma^{2}\right)}{6 \gamma}\left(r-r_{h}\right)\right\} \\
& \Phi(r)=r \sqrt{-V_{0}} .
\end{aligned}
$$

There is a horizon at $r=r_{h}$. Primes always denote derivatives with respect to the radial coordinate. The spectral densities are given by

$$
\begin{aligned}
\rho^{\text {shear }} & =\frac{e^{4 A-B} h}{32 \pi i G_{5} \sqrt{-V_{0}}}\left(h_{12}^{*} h_{12}^{\prime}-h_{12}^{*^{\prime}} h_{12}\right) \\
\rho^{\text {bulk }} & =\frac{e^{4 A-B} h \sqrt{-V_{0}}}{32 \pi i G_{5} A^{\prime 2}}\left(h_{11}^{*} h_{11}^{\prime}-h_{11}^{*^{\prime}} h_{11}\right) .
\end{aligned}
$$

The above quantities are independent of $r$; the right hand side can be evaluated at any value of $r$. The perturbations $h_{12}$ and $h_{11}$ are found by solving the linearized Einstein equations with the incoming wave boundary condition. These equations are:

$$
\begin{gathered}
h_{12}^{\prime \prime}(r)+h_{12}^{\prime}(r)\left[4 A^{\prime}(r)-B^{\prime}(r)+\frac{h^{\prime}(r)}{h(r)}\right] \\
+\left(\frac{w e^{B(r)-A(r)}}{h(r)}\right)^{2} h_{12}(r)=0,
\end{gathered}
$$

and,

[1] J. M. Maldacena, Adv. Theor. Math. Phys. 2, 231 (1998), arXiv:hep-th/9711200

[2] E. Witten, Adv. Theor. Math. Phys. 2, 253 (1998), arXiv:hep-th/9802150

[3] S. S. Gubser, I. R. Klebanov, and A. M. Polyakov, Phys. Lett. B428, 105 (1998), arXiv:hep-th/9802109.

[4] I. R. Klebanov and E. Witten, Nucl. Phys. B556, 89 (1999), arXiv:hep-th/9905104

[5] O. Aharony, S. S. Gubser, J. M. Maldacena, H. Ooguri, and Y. Oz, Phys. Rept. 323, 183 (2000), arXiv:hepth/9905111

[6] D. T. Son and A. O. Starinets, Ann. Rev. Nucl. Part. Sci. 57, 95 (2007), arXiv:0704.0240 [hep-th]

[7] J. Erdmenger, N. Evans, I. Kirsch, and E. Threlfall, Eur. Phys. J. A35, 81 (2008), arXiv:0711.4467 [hep-th].

[8] R. C. Myers and S. E. Vazquez, Class. Quant. Grav. 25, 114008 (2008), arXiv:0804.2423 [hep-th]

[9] S. S. Gubser and A. Karch, Ann. Rev. Nucl. Part. Sci. 59, 145 (2009), arXiv:0901.0935 [hep-th].

[10] I. R. Klebanov and A. A. Tseytlin, Nucl. Phys. B578, 123 (2000), arXiv:hep-th/0002159.

[11] I. R. Klebanov and M. J. Strassler, JHEP 08, 052 (2000),

$$
\begin{gathered}
h_{11}^{\prime \prime}(r)=\left[\frac{V_{0}}{3 A^{\prime}(r)}-4 A^{\prime}(r)+3 B^{\prime}(r)-\frac{h^{\prime}(r)}{h(r)}\right] h_{11}^{\prime}(r) \\
-\left[\left(\frac{w e^{B(r)-A(r)}}{h(r)}\right)^{2}+\frac{V_{0} h^{\prime}(r)}{6 h(r) A^{\prime}(r)}+\frac{h^{\prime}(r) B^{\prime}(r)}{h(r)}\right] h_{11}(r)
\end{gathered}
$$

These equations hold for any general single scalar gravity dual. The key observation is that for the Chamblin-Reall background,

$$
\frac{V_{0}}{3 A^{\prime}(r)}+2 B^{\prime}(r)=0
$$

With the use of this equality, one sees that the equation for $h_{12}$ becomes identical to that of $h_{11}$. Because the differential equations are the same, and the boundary conditions are the same, we conclude that for this background only, $h_{11}=h_{12}$, and thus, we again see that the bulk and shear spectral densities are simply related

$$
\frac{\rho^{\text {bulk }}(w)}{\rho^{\text {shear }}(w)}=-\frac{V_{0}}{A^{\prime}(r)^{2}}=9 \gamma^{2} .
$$

By comparing the definition of the potentials (48), (D1) we see that

Again we find

$$
\gamma^{2}=\frac{4 \delta}{3}
$$

$$
\frac{\rho^{\text {bulk }}(w)}{\rho^{\text {shear }}(w)}=12 \delta \text {. }
$$

This is in agreement with the result given in the text 57 .
arXiv:hep-th/0007191.

[12] K. Pilch and N. P. Warner, Nucl. Phys. B594, 209 (2001) arXiv:hep-th/0004063.

[13] M. Mia, K. Dasgupta, C. Gale, and S. Jeon, Nucl. Phys. B839, 187 (2010), arXiv:0902.1540 [hep-th]

[14] S. S. Gubser, S. S. Pufu, and F. D. Rocha, JHEP 08, 085 (2008), arXiv:0806.0407 [hep-th]

[15] S. S. Gubser and A. Nellore, Phys. Rev. D78, 086007 (2008), arXiv:0804.0434 [hep-th].

[16] U. Gursoy and E. Kiritsis, JHEP 02, 032 (2008) arXiv:0707.1324 [hep-th]

[17] U. Gursoy, E. Kiritsis, and F. Nitti, JHEP 02, 019 (2008), arXiv:0707.1349 [hep-th].

[18] U. Gursoy, E. Kiritsis, L. Mazzanti, and F. Nitti, JHEP 05, 033 (2009), arXiv:0812.0792 [hep-th].

[19] U. Gursoy, E. Kiritsis, G. Michalogiorgakis, and F. Nitti, JHEP 12, 056 (2009), arXiv:0906.1890 [hep-ph].

[20] T. Springer, Phys. Rev. D79, 046003 (2009) arXiv:0810.4354 [hep-th]

[21] T. Springer, Phys. Rev. D79, 086003 (2009) arXiv:0902.2566 [hep-th]

[22] B. Batell and T. Gherghetta, Phys. Rev. D78, 026002 
(2008), arXiv:0801.4383 [hep-ph].

[23] W. de Paula, T. Frederico, H. Forkel, and M. Beyer, Phys. Rev. D79, 075019 (2009), arXiv:0806.3830 [hep$\mathrm{ph}]$

[24] J. Alanen, K. Kajantie, and V. Suur-Uski, Phys. Rev. D80, 126008 (2009), arXiv:0911.2114 [hep-ph]

[25] J. Erlich, E. Katz, D. T. Son, and M. A. Stephanov, Phys. Rev. Lett. 95, 261602 (2005), arXiv:hep$\mathrm{ph} / 0501128$

[26] J. I. Kapusta and T. Springer, Phys. Rev. D81, 086009 (2010), arXiv:1001.4799 [hep-ph].

[27] P. Benincasa, A. Buchel, and A. O. Starinets, Nucl. Phys. B733, 160 (2006), arXiv:hep-th/0507026.

[28] O. Aharony, A. Buchel, and A. Yarom, Phys. Rev. D72, 066003 (2005), arXiv:hep-th/0506002.

[29] H. B. Meyer, Phys. Rev. D76, 101701 (2007), arXiv:0704.1801 [hep-lat]

[30] H. B. Meyer, JHEP 04, 099 (2010), arXiv:1002.3343 [heplat]

[31] D. Kharzeev and K. Tuchin, JHEP 09, 093 (2008), arXiv:0705.4280 [hep-ph]

[32] G. D. Moore and O. Saremi, JHEP 09, 015 (2008), arXiv:0805.4201 [hep-ph]

[33] A. Yarom, JHEP 04, 024 (2010), arXiv:0912.2100 [hepth]

[34] H. A. Chamblin and H. S. Reall, Nucl. Phys. B562, 133 (1999), arXiv:hep-th/9903225

[35] F. Bigazzi, A. L. Cotrone, and J. Tarrio, JHEP 02, 083 (2010), arXiv:0912.3256 [hep-th]

[36] S. S. Gubser, A. Nellore, S. S. Pufu, and F. D. Rocha, Phys. Rev. Lett. 101, 131601 (2008), arXiv:0804.1950 [hep-th]

[37] I. Kanitscheider and K. Skenderis, JHEP 04, 062 (2009), arXiv:0901.1487 [hep-th].
[38] P. Romatschke, Class. Quant. Grav. 27, 025006 (2010), arXiv:0906.4787 [hep-th].

[39] F. Bigazzi and A. L. Cotrone, JHEP 1008, 128 (2010), arXiv:arXiv:1006.4634 [hep-ph]

[40] P. Romatschke and D. T. Son, Phys. Rev. D80, 065021 (2009), arXiv:0903.3946 [hep-ph].

[41] T. Springer, C. Gale, S. Jeon, and S. H. Lee, Phys. Rev. D82, 106005 (2010), arXiv:1006.4667 [hep-th],

[42] G. Policastro, D. T. Son, and A. O. Starinets, JHEP 12, 054 (2002), arXiv:hep-th/0210220.

[43] P. K. Kovtun and A. O. Starinets, Phys. Rev. D72, 086009 (2005), arXiv:hep-th/0506184.

[44] J. Mas and J. Tarrio, JHEP 05, 036 (2007), arXiv:hepth/0703093

[45] D. Forster, Hydrodynamic fluctuations, broken symmetry, and correlation functions. (W. A. Benjamin, Inc.: Reading, Mass., Frontiers in Physics. Volume 47, 1975).

[46] L. G. Yaffe, Hydrodynamic Fluctuations in Relativistic Theories (unpublished, 1992).

[47] D. T. Son and A. O. Starinets, JHEP 09, 042 (2002), arXiv:hep-th/0205051.

[48] M. Kaminski, K. Landsteiner, J. Mas, J. P. Shock, and J. Tarrio, JHEP 02, 021 (2010), arXiv:0911.3610 [hepth]

[49] U. Gursoy, (2010), arXiv:1007.0500 [hep-th].

[50] Kapusta, J.I. and Gale, C., Finite-Temperature Field Theory: Principles and Applications (2nd edition, Cambridge Monographs on Mathematical Physics. Cambridge University Press, Cambridge, 2006).

[51] P. B. Arnold, C. Dogan, and G. D. Moore, Phys. Rev. D74, 085021 (2006), arXiv:hep-ph/0608012.

[52] P. Kovtun and A. Starinets, Phys. Rev. Lett. 96, 131601 (2006), arXiv:hep-th/0602059

[53] A. Buchel, J. T. Liu, and A. O. Starinets, Nucl. Phys. B707, 56 (2005), arXiv:hep-th/0406264. 